\title{
ARTIGO DE REVISÃO \\ Cuidado com vacinas na perspectiva evolucionária de Rodgers
}

\section{Care related to vaccines from the evolutionary perspective of Rodgers}

\author{
Suzane Gomes de Medeiros ${ }^{1}$ (D), Viviane Euzébia Pereira Santos² ${ }^{2}$ (D)
}

\section{RESUMO}

O estudo objetiva analisar o conceito cuidado com vacinas na perspectiva evolucionária de Rodgers. Refere-se a uma análise do conceito cuidado com vacinas, realizada nas bases de dados National Library of Medicine, Cumulative Index to Nursing and Allied Health Literature, Literatura Latino-Americana e do Caribe em Ciências da Saúde e Web of Science, com amostra final de 17 artigos, através de leitura crítico-reflexiva. Os atributos foram representados pelas propriedades e benefícios das vacinas. Os antecedentes referem-se aos efeitos do calor/congelamento/luz, perdas desnecessárias de vacinas, confiabilidade das vacinas comprometida. Os consequentes: necessidade de capacitação dos profissionais, segurança no estoque de vacinas e qualidade da imunização foram alguns elementos presentes. Os termos relacionados e as implicaçóes e hipóteses também foram determinados. O conceito do fenômeno proporciona subsídios para a promoção de práticas seguras e efetivas com vacinas e contribui para a construção de conhecimentos na enfermagem.

Descritores: Formação de Conceito; Enfermagem; Vacinas; Cuidados de Enfermagem.

\section{ABSTRACT}

The study aims to analyze the concept care related to vaccines from the evolutionary perspective of Rodgers. It refers to an analysis of the concept care related to vaccines, carried out in the National Library of Medicine databases, Cumulative Index to Nursing and Allied Health Literature, Latin-American and Caribbean on Health Sciences and Web of Science, with final sample of 17 articles, through critical and reflective reading. The attributes were represented by the properties and benefits of vaccines. The antecedents refer to heat/freeze/light effects, unnecessary losses of vaccines, compromised vaccines reliability. The resultants: need for the professionals' training, safety in the vaccines stock and quality of immunization were some elements presents. The terms related and the implications and hypotheses were also determined. The concept of the phenomenon provides grants for the promotion of safe and effective practices with vaccines and contributes to the construction of knowledge in the nursing area.

Descriptors: Concept Formation; Nursing; Vaccines; Nursing Care.

\footnotetext{
${ }^{1}$ Universidade do Estado do Rio Grande do Norte - Natal (RN), Brasil. E-mail:suzanegomesm@gmail.com

${ }^{2}$ Universidade Federal do Rio Grande do Norte - Natal (RN), Brasil. E-mail: vivianeepsantos@gmail.com

Como citar este artigo: Medeiros SG, Santos VEP. Cuidado com vacinas na perspectiva evolucionária de Rodgers. Rev. Eletr. Enferm. [Internet]. 2019 [acesso em: ];21:54309. Disponível em: https://doi.org/10.5216/ree.v21.54309.
}

Recebido em: 05/08/2018. Aceito em: 25/04/2018. Publicado em: 20/09/2019. 


\section{INTRODUÇÃO}

Ação subjetiva reconhecida universalmente, o cuidado está presente em todas as culturas. Entretanto, a diversidade de definiçóes relacionadas a esse termo é reflexo das práticas socioculturais e valores particulares existentes em cada grupo social e não apenas da realização de técnicas e procedimentos ou como ofício de uma profissão ${ }^{(1)}$.

$\mathrm{Na}$ área da saúde, o cuidado é considerado a essência da profissão de enfermagem. Esse ato é entendido como um elemento fundamental à sociedade e, para o seu pleno desempenho, exige produçáo de conhecimentos e competências especiais que embasem a atuação dos sujeitos que o realizam ${ }^{(2)}$.

A ação do cuidado é utilizada pelo enfermeiro para alcançar um fim, efetivada a partir de habilidades técnicas e saberes teóricos. Dentre as suas diversas atribuiçóes no cotidiano dos serviços, esse trabalhador é responsável por organizar, coordenar, planejar e avaliar as atividades com vacinas ${ }^{(2,3)}$.

Também denominadas imunobiológicos, as vacinas são preparaçóes que contêm micro-organismos vivos, mortos ou suas fraçôes, com propriedades antigênicas. $\mathrm{O}$ procedimento de administração das vacinas, através da introdução no organismo de antígenos ou agentes vivos atenuados, que induzem imunização, é chamado vacinação(4).

A imunização é caracterizada como o processo que leva à imunidade. Esta, por sua vez, corresponde ao estado de resistência associado à presença de anticorpos com uma ação peculiar sobre agentes causadores de doenças infecciosas ou sobre suas toxinas. Para que a imunização aconteça de forma plena e com segurança, deve ser acompanhada de cuidados, com açôes adequadas antes, durante e após a administração dos imunobiológicos ${ }^{(4)}$.

Por constituírem artigos termolábeis e fotossensíveis, as vacinas são facilmente afetadas pelas alterações de temperatura e luminosidade, respectivamente. Quando expostas às condiçôes que provocam desvio na sua qualidade, como o armazenamento inadequado, podem ter sua potência/eficácia e propriedades imunizantes comprometidas ${ }^{(4,5)}$.

Outros fatores podem cooperar para situaçóes que afetam o cuidado com vacinas, a exemplo da não adesão dos trabalhadores da enfermagem à prática do controle de temperatura, com influência na credibilidade dos imunobiológicos, no desperdício de recursos e prejuízos na cobertura vacinal. Ante esses problemas, estratégias foram idealizadas na perspectiva de mitigar falhas com esses produtos. Como uma das medidas, cita-se a criaçáo do Programa Nacional de Imunização (PNI), considerado referência mundial e com o propósito de preconizar a correta manipulação desses insumos ${ }^{(6,7)}$.

Em meio a esse contexto e em virtude dos diversos significados de cuidado que permeiam as práticas profissionais, vislumbra-se a necessidade de melhor desvelar as interfaces da expressão cuidado com vacinas, que requer clarificação e refinamento, uma vez que o uso inapropriado de alguns termos pode afetar essa prática.

Portanto, entende-se que é importante conceituá-la adequadamente para poder contribuir com a qualidade do cuidado. Para isso, é necessário aprofundar a compreensão sobre a temática, a partir da análise das publicaçóes nacionais e internacionais acerca desse conceito, o que permitirá contribuir com pesquisas futuras nessa área e colaborar com as práticas que envolvem a imunização.

Assim, este estudo será norteado pelo seguinte questionamento: Como o conceito cuidado com vacinas é utilizado nas pesquisas produzidas na área da saúde? Destarte, para responder a essa questáo, objetiva-se analisar o conceito cuidado com vacinas na perspectiva evolucionária de Rodgers ${ }^{(8)}$.

\section{MÉTODO}

Estudo desenvolvido a partir do método evolucionário de Rodgers, que representa um modelo indutivo, descritivo e não sequencial aplicado para averiguar a concordância e o conhecimento de um termo específico ao longo do tempo. Entende o conceito de forma dinâmica, através de um processo cíclico, em que o fenômeno estudado sofre modificaçóes com o transcorrer dos anos a partir de uma relaçáo direta com a conjuntura na qual é utilizado ${ }^{(8)}$. Esse método compreende seis etapas (Figura 1).

Nesta pesquisa, a definiçáo do conceito de interesse foi cuidado com vacinas, a partir das produçóes indexadas sobre o tema e suas expressóes associadas, referente a etapa 1. A segunda, a seleção do campo para a coleta de dados, ocorreu em dezembro de 2018, nas bases National Library of Medicine (PubMed), Cumulative Index to Nursing and Allied Health Literature (CINAHL), Literatura Latino-Americana e do Caribe em Ciências da Saúde (LILACS) e Web of Science, por possuírem materiais de qualidade científica e de visibilidade para a área da enfermagem.

Para nortear todo o trabalho, foi utilizado um protocolo elaborado pelas pesquisadoras, com indicadores de coleta, de forma a sistematizar o processo. $\mathrm{Na}$ caracterização das pesquisas incluíram-se: título, ano de publicação, local de estudo, tipo de estudo. $\mathrm{Na}$ análise os indicadores selecionados estão organizados de acordo com o método evolucionário de Rodgers $^{(8)}$ : atributos/conceito, base conceitual — antecedentes e consequentes, termos substitutos, termos relacionados, e implicações e hipóteses.

A seleçáo dos artigos ocorreu pela consulta aos Descritores em Ciências da Saúde (DeCS) e ao Medical Subject Headings $(\mathrm{MeSH})$, com seleção dos termos Atenção Primária à Saúde; Profissionais de Saúde e Cuidado com Vacinas para os DeCS, e Primary Health Care (\#1), Health Personnel (\#2), Care 
(\#3) e Vaccines (\#3) para os MeSH. Em todas as bases de dados inseridas na pesquisa foram adotados os cruzamentos: \#1 AND \#3, \#2 AND \#3 e \#1 AND \#2 AND \#3. O limite temporal das publicaçóes não foi utilizado, uma vez que se pretendeu desenvolver uma análise conceitual.

Para a terceira etapa, que se refere à coleta dos dados relevantes, com destaque dos atributos do conceito e bases conceituais, através dos antecedentes e consequentes, os

Figura 1. Desenvolvimento cíclico do conceito: as seis etapas do método evolucionário de Rodgers (2000). Natal/RN, 2019

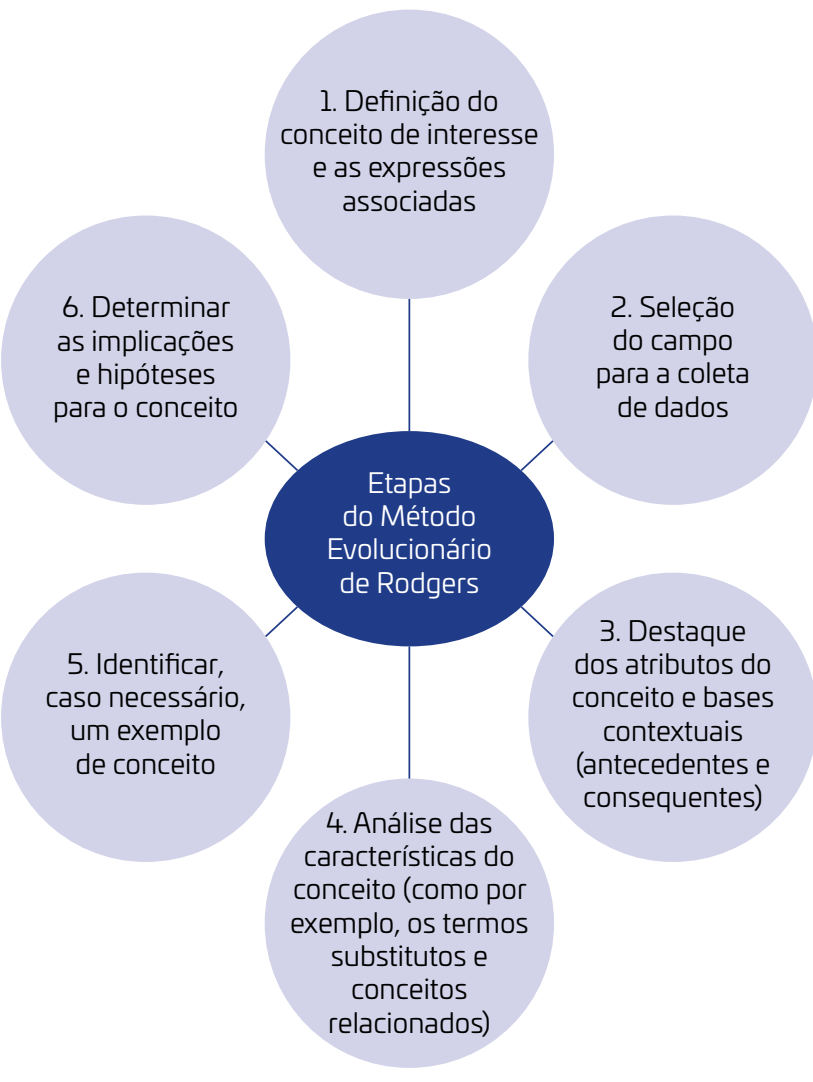

Fonte: adaptação de Rodgers (2000)(8). critérios de inclusão estabelecidos contemplaram: artigos científicos em português, espanhol e inglês que abordassem sobre a temática, disponíveis na íntegra eletronicamente. Os documentos repetidos foram analisados somente em uma base. Os demais materiais duplicados e que não estavam de acordo com a pesquisa foram excluídos.

A análise das características do conceito teve início com a leitura dos títulos dos trabalhos pré-selecionados na etapa 2, a partir dos critérios de elegibilidade. Esse processo foi necessário para selecionar os materiais de interesse para a pesquisa e extrair os elementos do modelo adotado, por meio de leitura objetiva.

Em seguida, foram identificados e separados nos textos os fragmentos que surgiram com mais frequência referente aos atributos, antecedentes e consequentes do conceito analisado. Os tópicos desses materiais localizados na literatura pesquisada foram digitados e, a partir de um procedimento indutivo, foram listados os possíveis atributos, antecedentes e consequentes.

Esses dados foram armazenados e inseridos em uma planilha do programa Microsoft Excel 2010', mediante dupla conferência, para organizar e facilitar a apreciaçáo subsequente. De acordo com o método de Rodgers ${ }^{(8)}$, a sua quinta etapa identifica um exemplo de conceito, caso seja necessário. Portanto, neste estudo, essa etapa não foi incluída em virtude de ser um item opcional. Para a sexta etapa foram determinadas as implicaçôes e hipóteses na perspectiva de contribuir com aspectos predominantes para a sucessão do conceito ao longo do tempo.

\section{RESULTADOS}

Foram encontrados 9.682 artigos. Após a leitura dos títulos e resumos e atendimento aos critérios de elegibilidade, foram excluídos 9.642, com pré-seleção de 40. Após refinamento, a amostra final contemplou $17^{(--25)}$ publicaçôes (Figura 2).

Todos os artigos foram lidos na íntegra, de maneira criteriosa, e distribuídos conforme autores, título e periódico, como demonstrado no Quadro 1. Dos selecionados, houve

Figura 2. Fluxograma de seleção dos artigos incluídos. Natal/RN, 2019.

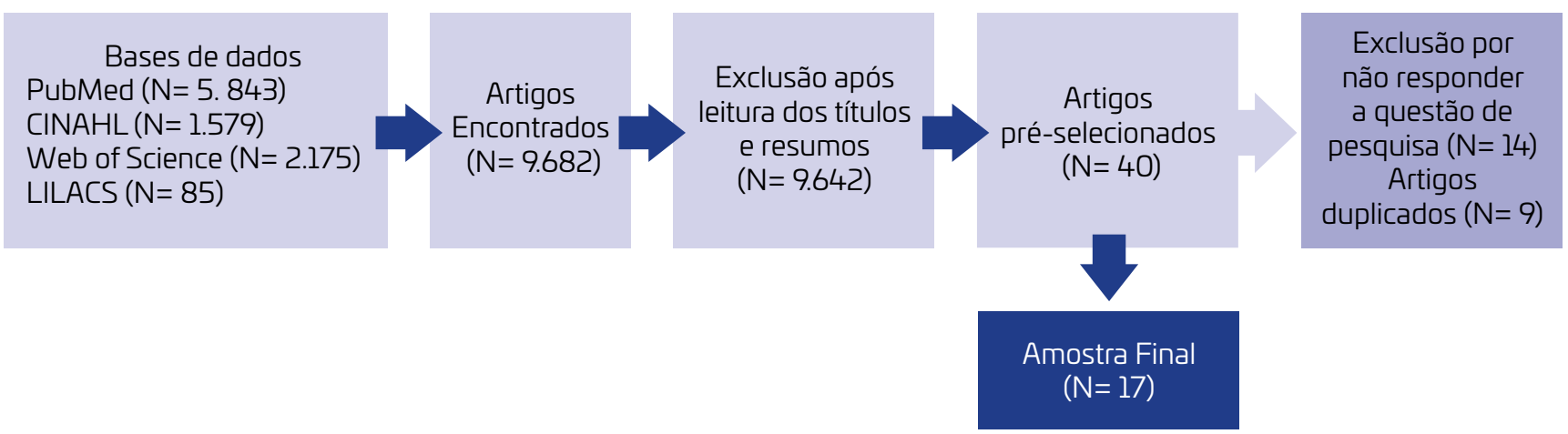


Quadro 1. Apresentação dos artigos incluídos na análise de conceito conforme autores, título e periódico.

\begin{tabular}{|c|c|c|}
\hline Autores & Título & Periódico \\
\hline $\begin{array}{l}\text { Barber-Hues C, Rodriguez-Sánchez } \\
\text { O, Cervera-Pérez I, Peiró S }\end{array}$ & $\begin{array}{c}\text { La cadena de frío vacunal en un departamento } \\
\text { de salud de la Comunidad Valenciana }{ }^{(9)}\end{array}$ & Gaceta Sanitaria \\
\hline $\begin{array}{l}\text { Oliveira VC, Gallardo MDPS, } \\
\text { Cavalcante RB, Arcêncio RA, Pinto IC }\end{array}$ & $\begin{array}{l}\text { Fragilidades da conservação de vacina nas } \\
\text { Unidades de Atenção Primária à Saúde }{ }^{(10)} \text {. }\end{array}$ & $\begin{array}{l}\text { Revista Brasileira de } \\
\text { Enfermagem }\end{array}$ \\
\hline $\begin{array}{l}\text { Oliveira VC, Guimarães EAA, } \\
\text { Guimarães IA, Januário LH, Pinto IC }\end{array}$ & $\begin{array}{l}\text { Prática da enfermagem na } \\
\text { conservação de vacinas(ll). }\end{array}$ & Acta Paulista de Enfermagem \\
\hline $\begin{array}{l}\text { Oliveira VC, Gallardo MDPS, } \\
\text { Arcêncio RA, Gontijo TL, Pinto IC }\end{array}$ & $\begin{array}{l}\text { Avaliação da qualidade de conservação de } \\
\text { vacinas na Atenção Primária à Saúde }{ }^{(12)} \text {. }\end{array}$ & Ciência e Saúde Coletiva \\
\hline $\begin{array}{l}\text { Mallik S, Mandal PK, } \\
\text { Chatterjee C, Ghosh P, } \\
\text { Manna N, Chakrabarty D, et al. }\end{array}$ & $\begin{array}{l}\text { Assessing cold chain status in a metro } \\
\text { city of India: an intervention study }{ }^{(13)} \text {. }\end{array}$ & African Health Sciences \\
\hline $\begin{array}{l}\text { Ferreira AV, Freitas PHB, } \\
\text { Viegas SMF, Oliveira VC }\end{array}$ & $\begin{array}{l}\text { Acesso à sala de vacinas da estratégia saúde } \\
\text { da família: aspectos organizacionais(14). }\end{array}$ & $\begin{array}{l}\text { Revista de Enfermagem } \\
\text { UFPE On Line }\end{array}$ \\
\hline $\begin{array}{l}\text { Thielmann A, Viehmann } \\
\text { A, Weltermann BM }\end{array}$ & $\begin{array}{l}\text { Effectiveness of a web-based education } \\
\text { program to improve vaccine storage conditions } \\
\text { in primary care (Keep Cool) study protocol } \\
\text { for a randomized controlled trial }{ }^{(15)} \text {. }\end{array}$ & Trials \\
\hline $\begin{array}{l}\text { Ferreira AV, Oliveira CF, } \\
\text { Guimarães EAA, Cavalcante } \\
\text { RB, Moraes JT, Oliveira VC }\end{array}$ & $\begin{array}{l}\text { Acesso à sala de vacinas nos serviços } \\
\text { de atenção primária à saúde }{ }^{(16)} \text {. }\end{array}$ & $\begin{array}{l}\text { Revista Eletrônica } \\
\text { de Enfermagem }\end{array}$ \\
\hline $\begin{array}{l}\text { Siqueira LG, Martins AMEBL, } \\
\text { Versiani CMC, Almeida LAV, } \\
\text { Oliveira CS, Nascimento JE, et al. }\end{array}$ & $\begin{array}{c}\text { Avaliação da organização e funcionamento das } \\
\text { salas de vacina na atenção primária à saúde em } \\
\text { Montes Claros, Minas Gerais, Brasil, } 2015^{(17)} \text {. }\end{array}$ & $\begin{array}{l}\text { Epidemiologia e } \\
\text { Serviços de Saúde }\end{array}$ \\
\hline $\begin{array}{l}\text { Raglione D, Bezerra GAM, } \\
\text { Lopes MH, Nerger MLBR, } \\
\text { Guimarães TC, Sartori AMC }\end{array}$ & $\begin{array}{l}\text { Avaliação da rede de frio para conservação } \\
\text { de vacinas em unidades básicas de } \\
\text { saúde das regiões Sul e Centro-Oeste do } \\
\text { município de São Paulo em 2011-2012(18). }\end{array}$ & $\begin{array}{l}\text { Epidemiologia e } \\
\text { Serviços de Saúde }\end{array}$ \\
\hline Vasconcelos KCE, Rocha AS, Ayres JA & $\begin{array}{l}\text { Avaliação normativa das salas de vacinas na } \\
\text { rede pública de saúde do Município de Marília, } \\
\text { Estado de São Paulo, Brasil, 2008-2009(19). }\end{array}$ & $\begin{array}{l}\text { Epidemiologia e } \\
\text { Serviços de Saúde }\end{array}$ \\
\hline $\begin{array}{l}\text { Ogboghodo EO, Omuemu } \\
\text { vO, Odijie O, Odaman OJ }\end{array}$ & $\begin{array}{l}\text { Cold chain management practices of } \\
\text { health care workers in primary health } \\
\text { care facilities in Southern Nigeria }{ }^{(20)} \text {. }\end{array}$ & $\begin{array}{l}\text { The Pan African } \\
\text { Medical Journal }\end{array}$ \\
\hline \begin{tabular}{|l} 
Oliveira VC, Rennó HMS, Santos YR, \\
Rabelo AFG, Gallardo MPS, Pinto IC \\
\end{tabular} & $\begin{array}{l}\text { Educação para o trabalho em sala de vacina: } \\
\text { percepção dos profissionais de enfermagem }{ }^{(21)} \text {. }\end{array}$ & $\begin{array}{l}\text { Revista de Enfermagem } \\
\text { do Centro-Oeste Mineiro }\end{array}$ \\
\hline Mugharbel KM, Wakeel SMA & $\begin{array}{l}\text { Evaluation of the availability of cold } \\
\text { chain tools and an assessment of health } \\
\text { workers practice in Dammam }{ }^{(22)} \text {. }\end{array}$ & $\begin{array}{l}\text { Journal of Family and } \\
\text { Community Medicine }\end{array}$ \\
\hline $\begin{array}{l}\text { Brandão RMS, Castro IO, Lins } \\
\text { JMM, Campos MEL, Andrade } \\
\text { MS, Guimarães MAS }\end{array}$ & $\begin{array}{l}\text { Factors related to the conservation of } \\
\text { vaccines in the basic health units } \\
\text { (23). }\end{array}$ & $\begin{array}{l}\text { Revista de Enfermagem } \\
\text { UFPE On Line }\end{array}$ \\
\hline Yuan L, Daniels S, Naus M, Brcic B & $\begin{array}{l}\text { Vaccine storage and handling. Knowledge and } \\
\text { practice in primary care physicians' offices }{ }^{(24)} \text {. }\end{array}$ & $\begin{array}{l}\text { The official Journal of } \\
\text { the College of Family } \\
\text { Physicians of Canada }\end{array}$ \\
\hline Efe E, Oncel S, Ozer ZC & $\begin{array}{l}\text { What do midwives in one region in } \\
\text { Turkey know about cold chain?(25). }\end{array}$ & Midwifery \\
\hline
\end{tabular}


predominância da língua portuguesa, com 10 documentos, seis na língua inglesa e um em espanhol.

Com relação ao ano, os materiais datavam de 1995 a 2017, e sete artigos utilizaram o tipo de estudo transversal $(41,1 \%)$. O Brasil foi o país que apresentou o maior quantitativo de trabalhos, com 10 materiais (58,8\%). A Espanha, Nigéria, Arábia Saudita, Canadá, Turquia, Alemanha e Índia tiveram uma publicação cada.

Dentro do contexto da prática de enfermagem com vacinas, na fase de análise é primordial reconhecer e explorar os atributos do conceito cuidado com vacinas, para elucidar a sua natureza e significado. Nela são identificadas as características do conceito que se apresentam com mais frequência quando este é descrito ou definido.

Com isso, as particularidades ou atributos são expressões utilizadas a partir de palavras que retratam o termo investigado e representam uma definição real, em objeção a uma definição nominal que apenas permuta uma expressão sinônima para outra. É o conjunto de atributos que permite identificar a situação postulada no conceito ${ }^{(8)}$. Conforme as leituras, os atributos do cuidado com vacinas podem ser representados pelas suas propriedades e benefícios (Figura 3).

Os antecedentes e os consequentes de um conceito estão relacionados aos aspectos socioculturais, temporais, situacionais da categoria profissional no atual contexto de análise ${ }^{(8)}$. Os antecedentes representam os fenômenos, situaçóes ou eventos que preexistem ao conceito analisado. Contribuem na compreensão do âmbito social no qual o conceito é usualmente utilizado, bem como auxiliam em seu refinamento. Assim, busca-se encontrar os episódios que favorecem o surgimento do termo cuidado com vacinas. Os consequentes são empregados para indicar ideias negligenciadas ou relaçóes pertinentes para novos caminhos de investigação ${ }^{(8)}$. Logo, os consequentes constituem os resultados da aplicação da expressão de interesse, a partir das implicações após a utilização desse mesmo termo (Figura 4).

A definição das expressóes substitutas consiste na aproximação inicial com o conceito de interesse, em que

Figura 3. Atributos do conceito cuidado com vacinas.

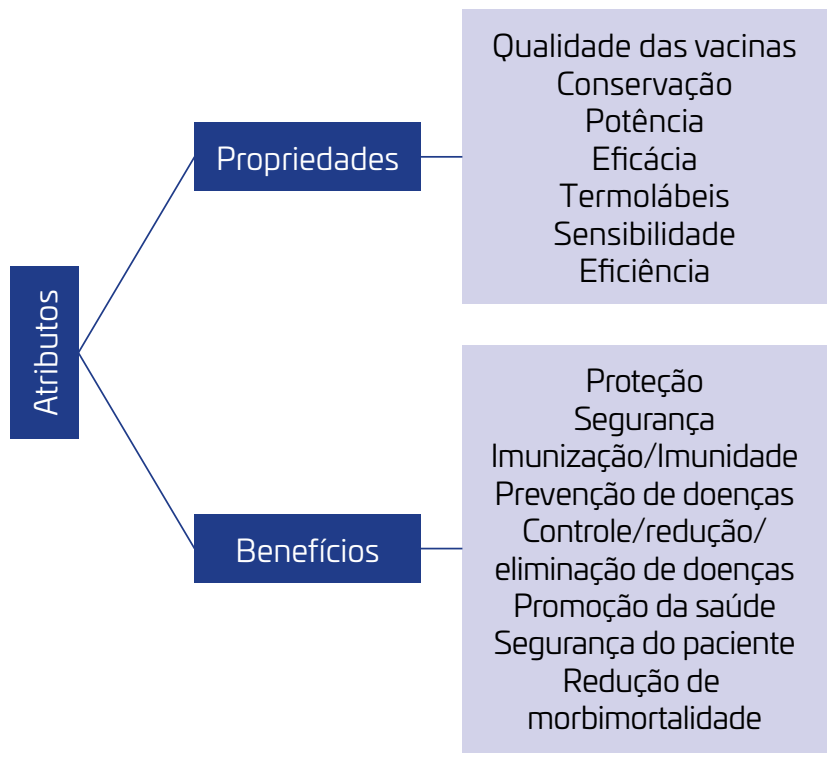

Figura 4. Antecedentes e consequentes do conceito cuidado com vacinas. Natal/RN, 2019.

- Efeitos do calor/congelamento/luz nas vacinas

- Perdas desnecessárias de vacinas

- Falta de acompanhamento e supervisão da sala de vacina pelo enfermeiro

- Aumento nos custos do PNI

- Procedimento inadequado no armazenamento, transporte ou manipulação;

- Entraves na conservação dos imunobiológicos

- Salas de vacinas com falhas na estrutura

- Confiabilidade das vacinas comprometida

- Falta de capacitação para os profissionais que trabalham nas salas de vacinas
- Necessidade de capacitação dos profissionais

- Segurança no estoque de vacinas

- Confiança da população na vacinação

- Prevenção/controle de doenças imunopreveníveis

- Garantia/manutenção da máxima potência e eficácia das vacinas

- Qualidade da imunização

- Cuidados com a conservação de vacinas para evitar perdas desnecessárias

- Redução de custos

- Registro diário da temperatura do refrigerador 
esses termos exprimem a forma de empregar o conceito de maneira diferente do termo ou palavra habitualmente usada nos materiais investigados ${ }^{(8)}$. Para isso, buscam-se os termos utilizados nos textos, de maneira a entender sua origem, desenvolvimento, interconexóes e funçóes do conceito com as várias palavras correspondentes.

As implicaçóes e hipóteses estabelecem, respectivamente, os resultados e possibilidades atingidos no cuidado com vacinas no contexto da enfermagem. São elementos inerentes para a evolução do conceito no decorrer do tempo e importantes para que novas pesquisas sejam desenvolvidas ${ }^{(8)}$.

\section{DISCUSSÃO}

\section{Cuidado com vacinas: atributos e conceitos}

As vacinas constituem produtos comparáveis em termos de eficácia, segurança e qualidade, e preservar suas propriedades ideais tem impacto de forma positiva nas condições de saúde da população, através da erradicação de doenças. Para que a imunidade e potência das vacinas sejam asseguradas é fundamental a manutenção da cadeia de frio (CF) de conservação desses insumos em todo o seu processo, da produção até a administração nas pessoas, de forma que sejam evitadas alteraçôes na sua potência e composição, uma vez que são sensíveis à luz, calor, umidade e congelamento ${ }^{(26-29)}$.

A manutenção da CF visa garantir que a população receba vacinas eficientes, com contribuição para o controle e erradicação de doenças imunopreveníveis. Esse fator contribui para a utilização segura desses insumos e reforça o cuidado de enfermagem nessa prática, além de favorecer a promoção da saúde ${ }^{(28,29)}$.

O conceito são as expressóes reais a respeito de um termo específico $^{(8)}$. Nos artigos selecionados, identifica-se uma escassez do conceito cuidado com vacinas, uma vez que não se encontra de forma explícita nas publicaçóes.

Embora a vacinação seja uma atividade conhecida, o conceito cuidado com vacinas não está claramente evidenciado nos artigos analisados. Essa ideia é abordada na literatura ao afirmar que o contexto que envolve o campo da vacinaçáo merece um olhar diferenciado, com maior produçáo de pesquisas nessa área por se tratar de um tema atual ${ }^{(30)}$.

Entretanto, após uma leitura detalhada, identifica-se em um dos materiais selecionados que o fenômeno investigado pode ser conceituado como os cuidados essenciais para garantir eficácia das vacinas ${ }^{(9)}$. Da mesma forma, para esse cuidado é indispensável um rígido controle das condiçóes de conservação de vacinas, para garantir a qualidade e a efetividade da imunização ${ }^{(10)}$. Essa opinião é reforçada por outro artigo ao enfatizar que os imunobiológicos necessitam de conservação adequada, o que requer instalaçôes, equipamentos e procedimentos de manipulação apropriados ${ }^{(17)}$.
Diante do exposto, o conceito analisado precisa ser ampliado, com inserção em sua formatação de outros elementos, também indispensáveis no cuidado com vacinas. A pesquisa revelou que esse conceito ainda está muito voltado para a conservação dos imunobiológicos. Para tanto, termos como promoção da saúde, segurança do paciente e qualificação profissional podem ser adicionados, de forma a contemplar as dimensóes da prática, dos profissionais e da população.

\section{Conceito cuidado com vacinas: antecedentes e consequentes}

Diante dos dados encontrados, entende-se que os antecedentes estáo relacionados aos aspectos que comprometem a manutenção da qualidade dos imunobiológicos. Um agravante nesse processo, considerado como evento crítico, é o fato de muitos profissionais não possuírem conhecimentos básicos sobre conservação de vacinas. Identifica-se, portanto, a necessidade de capacitação dos recursos humanos, bem como a supervisão desses profissionais na prática de imunização, para que o manejo com vacinas aconteça de acordo com as normas preconizadas pelo $\mathrm{PNI}^{(28)}$.

A possibilidade de carência nas atualizaçôes dos trabalhadores atuantes em sala de vacina favorece para que o cuidado com esses produtos seja prejudicado. Dessa forma, variaçôes na temperatura dos imunobiológicos consistem em um grave problema, com repercussão na sua potência e eficácia. É a partir da sucessão de pequenas falhas que a confiabilidade nessas substâncias diminui e os custos para o sistema de saúde aumentam, com perda desnecessária no estoque de insumos ou utilização desses sem efeito na população ${ }^{(28)}$.

Além disso, lacunas nos procedimentos de armazenamento, transporte e manipulação podem interferir na qualidade das vacinas, que se deterioram depois de determinado período, em razão da exposição à temperatura inapropriada à sua conservação. As questôes estruturais na sala de vacina também merecem atenção, pois são escassos trabalhos sobre a análise da infraestrutura nesses espaços ${ }^{(28,31)}$.

Todos esses fatores são indispensáveis no cuidado com vacinas. Dessa forma, os consequentes estão diretamente envolvidos com os antecedentes e, de acordo com a Figura 4, são resultantes da ocorrência do fenômeno investigado. Assim, surge a necessidade de intervençóes que permitam o desenvolvimento de um cuidado relacionado à prática vacinal, com valorização do papel desempenhado pela equipe de enfermagem ${ }^{(30)}$.

Para isso, é importante investir em atualização dos profissionais, pois os conhecimentos em vacinação estão em constante transformação. Assim, as capacitaçóes representam uma conduta que favorece a segurança das vacinas, com aumento de sua credibilidade e consequente adesão da população. Esse processo de qualificação favorece práticas 
seguras em imunização no cotidiano dos serviços, com benefícios relativos ao controle de doenças imunopreveníveis e consequente melhoria da saúde da populaçãoo ${ }^{(30,31)}$.

Os resultados efetivos da vacinaçáo segura estáo condicionados a alguns aspectos indispensáveis, como trabalhadores devidamente capacitados, conservação e manutenção da temperatura apropriada dos imunobiológicos, de forma que o controle de qualidade do estoque seja mantido em seu estado de potência máxima, para garantir a segurança do paciente nesse processo. Estudo brasileiro realizado a partir de revisão integrativa aponta algumas medidas para evitar que a estabilidade e potência das vacinas sejam alteradas, dentre elas identifica a produção de vacinas termoestáveis, mais robustas às condiçóes adversas ao calor, e recomenda adotar dispositivos para monitorar as exposiçóes desses produtos a temperaturas variadas ${ }^{(28)}$.

Diante disso, algumas recomendaçôes têm sido enfatizadas para evitar a perda desnecessária de vacinas, uma vez que o prejuízo no estoque desses artigos é bastante oneroso ao sistema. Tal necessidade enfoca a importância de garantir que as condiçóes de armazenamento desses produtos sejam cumpridas com a finalidade de assegurar a qualidade das vacinas, em seu estado de potência máxima ${ }^{(28,31)}$.

Nessa perspectiva, as normas preconizadas pelo PNI devem ser adotadas para evitar que os imunobiológicos se estraguem, ou ocorra deficiência na proteção dos indivíduos. Contudo, assegurar a qualidade das vacinas ainda constitui um desafio para os profissionais de saúde. Entretanto, para a saúde pública, os imunobiológicos simbolizam uma excelente estratégia de intervenção e mantêm uma das melhores relações custo-benefício até hoje adotadas ${ }^{(27)}$.

\section{Termos relacionados ao cuidado com vacinas}

Para cuidado com vacinas os termos substitutos e os respectivos estudos nos quais foram localizados são: Preservar/conservar vacinas/imunobiológicos ${ }^{(9-11,21)}$; Práticas adequadas no processo de conservação ${ }^{(10,11,17,19,22,23)}$; Manutenção da segurança de estoque ${ }^{(13)}$; Qualidade de conservaçáo das vacinas ${ }^{(12,21)}$; Qualidade do cuidado em sala de vacina ${ }^{(14,21)}$; Qualidade da assistência prestada no serviço $^{(10,14,17,20,21)}$; e Serviço de imunização de qualidade ${ }^{(21)}$.

Esses termos substitutos do cuidado com vacinas são expressóes com sentidos semelhantes, identificadas com base na leitura dos artigos selecionados. Dessa forma, percebe-se que o termo mais frequente está relacionado às práticas adequadas no processo de conservação, presente em seis materiais pesquisados.

Os conceitos relacionados são suposiçóes que se referem a outros conceitos trabalhados em associação ao de interesse. Possibilitam uma proximidade com o conceito investigado, mas não são sinônimos, pois adquire um novo significado ${ }^{(8)}$.
No cuidado com vacinas, os conceitos relacionados são formulados por dois agrupamentos: 1) suposiçóes estruturais e organizacionais das salas de vacina; 2) suposiçóes de educação e condutas com os imunobiológicos.

Para o agrupamento 1, os conceitos relacionados e os respectivos estudos nos quais foi possível sua identificação são: Qualidade na conservação e estocagem dos imunobiológicos ${ }^{(12,13,17,19,20,23)}$; Organização interna dos refrigeradores $^{(13,18,19,23)}$; Limpeza e degelo adequado dos equipamentos $^{(11,16-18,23)}$; Conhecimento, manutenção e gestão da Cadeia de Frio ${ }^{(9,13,15,16,20)}$; Monitoramento, registro diário e notificação de alteraçôes na temperatura ${ }^{(11,13,15,17,19)}$; Atenção rigorosa no armazenamento, transporte e manuseio ${ }^{(10,17,19,22)}$; Cumprimento das recomendaçóes do $\mathrm{PNI}^{(10,17)}$.

Em relaçáo ao agrupamento 2, foram encontrados: Açôes educativas ${ }^{(10,17,19-21,23)}$; Investimentos na formação dos profissionais que atuam em sala de vacina ${ }^{(11,20,21)}$; Supervisão desses espaços pelo enfermeiro ${ }^{(11,12,14,17-23)}$; Açóes de vigilância ${ }^{(17,19)}$; Qualidade das vacinas ${ }^{(10-12,19,20,23)}$; Controle das atividades que envolvem manipulação das vacinas ${ }^{(10,17)}$; Estabelecer protocolos de ação ${ }^{(9,18)}$.

Dessa forma, as suposiçóes operacionais e organizacionais das salas de vacina, presentes no agrupamento 1 , envolvem a qualidade na conservação e estocagem dos imunobiológicos através da organizaçáo interna dos refrigeradores, bem como a limpeza e degelo adequado desses equipamentos. Assim, são elementos essenciais para o cuidado com vacinas: o conhecimento, manutenção e gestão da Cadeia de Frio; o monitoramento, registro diário e notificaçáo de alteraçôes na temperatura; atenção rigorosa no armazenamento, transporte e manuseio; cumprimento das recomendaçóes técnicas do PNI.

As suposiçóes de educação e condutas com os imunobiológicos, evidenciadas no agrupamento 2, reforçam a necessidade de melhores práticas para assegurar a potência ideal das vacinas, através de açóes educativas, com mais investimentos na formação dos profissionais que atuam em sala de vacina. Para isso, a supervisão desses espaços pelo enfermeiro representa uma das prioridades em virtude de a prática de imunização ser uma competência da enfermagem.

Para essa suposição, encontra-se também a importância de açóes de vigilância para reforçar a qualidade das vacinas, com controle das atividades que envolvem a manipulação das vacinas. Dessa forma, estabelecer protocolos de ação influencia positivamente no cuidado com vacinas e constitui um eixo basilar para a segurança com esses produtos.

\section{Implicações e hipóteses do conceito cuidado com vacinas}

A partir dos materiais selecionados, esses termos estão especificados e acompanhados do número do estudo no qual foram identificados. Com isso, as implicações e hipóteses são: Garantia da qualidade e/ou conservação das vacinas ${ }^{(10-12,14)}$; 
Processo de educação para o trabalho em sala de vacina de forma contínua ${ }^{(21,22)}$; Profissionais capacitados ${ }^{(11,12,14,16,18,21,23)}$; Qualidade e/ou segurança das vacinas ${ }^{(14,19,22,23)}$; Eficiência, eficácia e efetividade na conservação das vacinas ${ }^{(10)}$; Garantia de uma assistência de qualidade em sala de vacina ${ }^{(14)}$.

Percebe-se que o cuidado com vacinas pode representar uma ferramenta encorajadora para o aperfeiçoamento e o exercício seguro da utilizaçáo desses produtos ${ }^{(10)}$. Esse contexto necessita que o enfermeiro estabeleça prioridades de atuaçáo e maior participação nas açóes de capacitação da sua equipe, de forma a contribuir para a qualidade da assistência, diretamente relacionada ao conhecimento dos profissionais e gerenciamento do cuidado ${ }^{(30)}$.

Nessa perspectiva, é preciso compreender esse cuidado de forma contínua, dinâmica e de constante aperfeiçoamento para os profissionais envolvidos. Para isso, esses trabalhadores necessitam ter capacidade de adaptação às mudanças, através de atualização de seus saberes, mediados por treinamentos e reciclagem da equipe ${ }^{(10)}$. Todo esse processo é importante em razão da introdução de novas vacinas no calendário vacinal e acréscimo das faixas etárias com recomendação de vacinação ${ }^{(30)}$. Portanto, esses elementos constituem medidas estratégicas para as atividades de imunização presentes na prática da enfermagem.

Com isso, acredita-se na hipótese de que o cuidado com vacinas requer atualizaçóes periódicas pelos profissionais da enfermagem, de forma a favorecer a eficácia e segurança desses insumos. É possível que os treinamentos contribuam para aperfeiçoar o processo de trabalho no ambiente de sala de vacina, a qualidade de conservação dos produtos e promover a satisfação dos pacientes.

Portanto, os termos encontrados neste estudo fornecem implicaçóes para o cuidado com vacinas, uma vez que a enfermagem fortalece saberes através da compreensão de conceitos de maneira ampliada e sua correta utilização. Entende-se a importância e continuidade de pesquisas futuras para o delineamento do conceito cuidado com vacinas com o propósito de refinar investigaçóes para aplicação e o uso desse conceito pela enfermagem.

Como forma de esquematizar o que foi discutido no estudo, uma síntese dos termos associados ao cuidado com vacinas foi elaborada (Figura 5).

Figura 5. Síntese dos termos associados ao conceito de cuidado com vacinas.

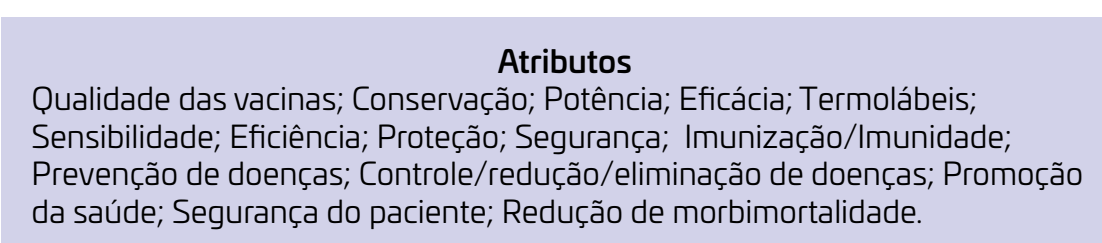

\section{Antecedentes}

Efeitos do congelamento; Perdas desnecessárias; Falta de acompanhamento e supervisão pelo enfermeiro; Aumento nos custos do PNI; Procedimento inadequado no armazenamento, transporte ou manipulação; Entraves na conservação; Falhas na estrutura; Confiabilidade das vacinas comprometida; Falta de capacitação para os profissionais.

\section{Implicações e Hipóteses}

Qualidade de conservação; Eficácia e Segurança; Satisfação dos pacientes; Melhoria no processo de trabalho; Profissionais capacitados.

\section{Consequentes}

Necessidade de capacitação; Segurança no estoque; Confiança da população na vacinação; Prevenção/controle de doenças; Garantia da máxima potência e eficácia; Qualidade da imunização; Cuidados com a conservação para evitar perdas desnecessárias; Redução de custos; Registro diário da temperatura.

\section{Termos substitutos}

Preservar/conservar vacinas/imunobiológicos; Práticas corretas; Manutenção das condições ideais das vacinas; Qualidade de conservação; Cuidado em sala de vacina.

\section{Conceitos relacionados}

Qualidade na conservação e estocagem; Disposição correta nos refrigeradores; Limpeza e degelo adequado dos equipamentos; Conhecimento, manutenção e gestão da Cadeia de Frio; Monitoramento, registro diário e notificação de alterações na temperatura; Atenção rigorosa no armazenamento, transporte e manuseio; Cumprimento das recomendações do PNl; Processo educativo; Investimentos na formação dos profissionais; Supervisão pelo enfermeiro; Ações de vigilância; Segurança dos procedimentos; Qualidade das vacinas; Avaliação das atividades; Estabelecer protocolos de ação. 


\section{CONCLUSÃO}

A partir do Método Evolucionário de Rodgers, nota-se que a análise do conceito cuidado com vacinas foi mais bem compreendida, embora ainda ocorra a necessidade de mais investigaçóes. Esse detalhamento acerca do fenômeno em questấo pode contribuir para a assistência de enfermagem com vacinas ao estabelecer açóes mais efetivas.

Desse modo, com base nos resultados encontrados, a identificação dos termos permite a formulação do conceito cuidado com vacinas: se trata de uma intervenção caracterizada como um processo contínuo, edificado através de aspectos qualificadores para manutenção da potência e eficácia dos imunobiológicos que envolvem dimensóes gerenciais, organizacionais, infraestruturais, e apresenta relação com a necessidade de adaptação às mudanças, com introdução de novos hábitos, provenientes de atualizaçóes dos profissionais de enfermagem, que viabilizem condutas responsáveis e seguras para conservação das vacinas, na perspectiva de garantir benefícios à população em geral.

Em face do exposto, a análise de conceito, apesar de complexa, contribui para o suporte e consolidação de conhecimento na enfermagem, com subsídios para a promoção de práticas seguras, assim como novas reflexôes acerca do cuidado com vacinas.

\section{REFERÊNCIAS}

1. Salbego C, Dornelles CS, Greco PBT, Pradebon VM, Alberti GF. Significado do cuidado para enfermagem de centro cirúrgico. Rev Rene [Internet]. 2015 [acessado em 3 dez. 2018];16(1):46-53. Disponível em: http://www. revistarene.ufc.br/revista/index.php/revista/article/ viewFile/1893/pdf. $\quad$ http://doi.org/10.15253/21756783.2015000100007.

2. Thofehrn MB, Montesinos MJL, Arrieira IC, Àvila VC, Vasques TCS, Farias ID. Nurses' work process in a hospital in spain: emphasis on the technologies of care. Cogitare Enferm [Internet]. 2014 [acessado em 3 dez. 2018];19(1):141-6. Disponível em: http://revistas.ufpr. br/cogitare/article/view/35972/22180.

3. Fossa AM, Protti AM, Rocha MCP, Horibe TM, Pedroso GER. Conservação e administração de vacinas: a atuação da enfermagem. Saúde Rev [Internet]. 2015 [acessado em 3 dez. 2018];15(40):85-96. Disponível em: $\quad$ https://www.metodista.br/revistas/revistasunimep/index.php/sr/article/view/2538/1475. http:// dx.doi.org/10.15600/2238-1244/sr.v15n40p85-96.

4. Brasil. Ministério da Saúde. Manual de Normas e Procedimentos para Vacinação. Brasília: Ministério da Saúde; 2014.

5. Oliveira VC, Caveião C, Crosewski F. Nursing management in the control of avoidable losses of immunobiological preparations. Cogitare Enferm [Internet]. 2014 [acessado em 12 dez. 2018];19(4):679-86. Disponível em: http://revistas. ufpr.br/cogitare/article/view/36358/23921.

6. Pereira DDS, Neves EB, Gemelli M, Ulbricht L. Análise da taxa de utilizaçáo e perda de vacinas no programa nacional de imunização. Cad Saúde Colet [Internet]. 2013 [acessado em 20 dez. 2018];21(4):420-4. Disponível em: http://www.scielo.br/pdf/cadsc/v21n4/v21n4a10.pdf. http://dx.doi.org/10.1590/S1414-462X2013000400010.

7. Giovelli G, Cardoso SMM, Fontana RT, Rodrigues FCP, Brum ZP. Nursing technicians' perceptions regarding the occupational risks in vaccination rooms. Cogitare Enferm [Internet]. 2014 [acessado em $20 \mathrm{dez}$. 2018];19(2):330-6. Disponível em: http://revistas.ufpr. br/cogitare/article/viewFile/37006/22814.

8. Rodgers BL. Concept Analysis: an evolutionary. In: Rodgers BL, Knafl KA, editores. Concept development in nursing: foundations, techniques, and applications. Filadélfia: Saunders; 2000. p. 77-102.

9. Barber-Hues C, Rodriguez-Sánchez O, Cervera-Pérez I, Peiró S. La cadena de frío vacunal en un departamento de salud de la Comunidad Valenciana. Gac Sanit [Internet]. 2009 [acessado em 20 dez. 2018];23(2):139-43. Disponível: http://scielo.isciii.es/pdf/gs/v23n2/breve1. pdf. http://dx.doi.org/10.1016/j.gaceta.2008.03.003.

10. Oliveira VC, Gallardo MDPS, Cavalcante RB, Arcêncio RA, Pinto IC. Fragilidades da conservação de vacina nas Unidades de Atenção Primária à Saúde. Rev Bras Enferm [Internet]. 2015 [acessado em 20 dez. 2018];68(2):291-6. Disponível: http://www.scielo.br/ pdf/reben/v68n2/0034-7167-reben-68-02-0291.pdf. http://dx.doi.org/10.1590/0034-7167.2015680215i.

11. Oliveira VC, Guimarães EAA, Guimarães IA, Januário LH, Pinto IC. Prática da enfermagem na conservaçáo de vacinas. Acta Paul Enferm [Internet]. 2009 [acessado em 20 dez. 2018];22(6):814-8. Disponível em: http:// www.scielo.br/pdf/ape/v22n6/a14v22n6.pdf. http:// dx.doi.org/10.1590/S0103-21002009000600014.

12. Oliveira VC, Gallardo MDPS, Arcêncio RA, Gontijo TL, Pinto IC. Avaliação da qualidade de conservação de vacinas na Atenção Primária à Saúde. Ciênc Saúde Coletiva [Internet]. 2014 [acessado em 20 dez. 2018];19(9):3889-98. Disponível em: http://www.scielo. br/pdf/csc/v19n9/1413-8123-csc-19-09-3889.pdf. http://doi.org/10.1590/1413-81232014199.12252013.

13. Mallik S, Mandal PK, Chatterjee C, Ghosh P, Manna $\mathrm{N}$, Chakrabarty D, et al. Assessing cold chain status in a metro city of India: an intervention study. Afr Health Sci [Internet]. 2011 [acessado em 20 dez. 2018];11(1):128-33. Disponível em: https://www.ncbi.nlm.nih.gov/pmc/ articles/PMC3092313/pdf/AFHS1101-0128.pdf. 
14. Ferreira AV, Freitas PHB, Viegas SMF, Oliveira VC. Acesso à sala de vacinas da estratégia saúde da família: aspectos organizacionais. Rev Enferm UFPE on line [Internet]. 2017 [acessado em $20 \mathrm{dez}$. 2018];11(10):3869-77. Disponível em: https:// periodicos.ufpe.br/revistas/revistaenfermagem/article/ viewFile/69709/24321. https://doi.org/10.5216/ree. v19.42468.

15. Thielmann A, Viehmann A, Weltermann BM. Effectiveness of a web-based education program to improve vaccine storage conditions in primary care (Keep Cool) study protocol for a randomized controlled trial. Trials [Internet]. 2015 [acessado em 20 dez. 2018];16:301-9. Disponível em: https:// www.ncbi.nlm.nih.gov/pmc/articles/PMC4501106/ pdf/13063 2015 Article 824.pdf. $\underline{\text { https://doi. }}$ org/10.1186/s13063-015-0824-9.

16. Ferreira AV, Oliveira CF, Guimarães EAA, Cavalcante RB, Moraes JT, Oliveira VC. Acesso à sala de vacinas nos serviços de atenção primária à saúde. Rev Eletr Enf [Internet]. 2017 [acessado em 20 dez. 2018];19:a31. Disponível em: https://www.revistas.ufg.br/fen/article/ view/42468/24010. http://dx.doi.org/10.5216/ree. v19.42468.

17. Siqueira LG, Martins AMEBL, Versiani CMC, Almeida LAV, Oliveira CS, Nascimento JE, et al. Avaliaçáo da organização e funcionamento das salas de vacina na atençâo primária à saúde em Montes Claros, Minas Gerais, Brasil, 2015. Epidemiol Serv Saúde [Internet]. 2017 [acessado em 20 dez. 2018];26(3):557-68. Disponível em: http://www.scielo.br/pdf/ress/ v26n3/2237-9622-ress-26-03-00557.pdf. http://doi. org/10.5123/S1679-49742017000300013.

18. Raglione D, Bezerra GAM, Lopes $\mathrm{MH}$, Nerger MLBR, Guimarães TC, Sartori AMC. Avaliação da rede de frio para conservação de vacinas em unidades básicas de saúde das regióes Sul e Centro-Oeste do município de São Paulo em 2011-2012. Epidemiol Serv Saúde [Internet]. 2016 [acessado em $20 \mathrm{dez}$. 2018];25(1):65-74. Disponível em: http://www.scielo. br/pdf/ress/v25n1/2237-9622-ress-25-01-00065.pdf. http://doi.org/10.5123/S1679-49742016000100007.

19. Vasconcelos KCE, Rocha AS, Ayres JA. Avaliação normativa das salas de vacinas na rede pública de saúde do Município de Marília, Estado de São Paulo, Brasil, 2008-2009. Epidemiol Serv Saúde [Internet]. 2012 [acessado em 20 dez. 2018];21(1):167-76. Disponível em: http://scielo.iec.gov.br/pdf/ess/ v21n1/v21n1a17.pdf. http://doi.org/10.5123/S167949742012000100017.

20. Ogboghodo EO, Omuemu VO, Odijie O, Odaman OJ. Cold chain management practices of health care workers in primary health care facilities in Southern Nigeria. Pan Afr Med J. [Internet]. 2017 [acessado em 20 dez. 2018];27:34. Disponível em: $\quad$ https://www.ncbi.nlm.nih.gov/pmc/articles/ PMC5516677/pdf/PAMJ-27-34.pdf. https://dx.doi. org/10.11604\%2Fpamj.2017.27.34.11946.

21. Oliveira VC, Rennó HMS, Santos YR, Rabelo AFG, Gallardo MPS, Pinto IC. Educação para o trabalho em sala de vacina: percepção dos profissionais de enfermagem. R Enferm Cent O Min [Internet]. 2016 [acessado em 20 dez. 2018];6(3):2331-41. Disponível em: http://www.seer. ufsj.edu.br/index.php/recom/article/view/1180/1166. http://doi.org/10.19175/recom.v6i3.1180.

22. Mugharbel KM, Wakeel SMA. Evaluation of the availability of cold chain tools and an assessment of health workers practice in Dammam. J Fam Community Med [Internet]. 2009 [acessado em 20 dez. 2018];16(3):83-8. Disponível em: https://www. ncbi.nlm.nih.gov/pmc/articles/PMC3377047.

23. Brandão RMS, Castro IO, Lins JMM, Campos MEL, Andrade MS, Guimarães MAS. Factors related to the conservation of vaccines in the basic health units. Rev Enferm UFPE on line [Internet]. 2012 [acessado em 20 dez. 2018];6(2):332-8. Disponível em: https:// periodicos.ufpe.br/revistas/revistaenfermagem/article/ view/7034/6287.

24. Yuan L, Daniels S, Naus M, Brcic B. Vaccine storage and handling. Knowledge and practice in primary care physicians' offices. Can Fam Physician [Internet]. 1995 [acessado em 20 dez. 2018];41:1169-76. Disponível em: $\quad$ https://www.ncbi.nlm.nih.gov/pmc/articles/ PMC2146189/pdf/canfamphys00089-0039.pdf.

25. Efe E, Oncel S, Ozer ZC. What do midwives in one region in Turkey know about cold chain?. Midwifery [Internet]. 2008 [acessado em 20 dez. 2018];24(3):32834. Disponível em: https://doi.org/10.1016/j. midw.2006.11.002.

26. Barata-Silva C, Hauser-Davis RA, Silva ALO, Moreira JC. Desafios ao controle da qualidade de medicamentos no Brasil. Cad Saúde Col [Internet]. 2017 [acessado em 29 dez. 2018];25(3):362-70. Disponível em: http://www.scielo.br/pdf/cadsc/v25n3/1414-462Xcadsc-1414-462X201700030075.pdf. $\underline{\text { http://doi. }}$ org/10.1590/1414-462X201700030075.

27. Crosewski F, Larocca LM, Chaves MMN. Perdas evitáveis de imunobiológicos na instância local: reflexóes acerca do processo de trabalho da enfermagem. Saúde Debate [Internet]. 2018 [acessado em 29 dez. 2018];42(116):203-13. Disponível em: http://www.scielo.br/pdf/sdeb/v42n116/0103-1104sdeb-42-116-0203.pdf. http://doi.org/10.1590/01031104201811616. 
28. Guimarães EAA, Oliveira VC, Oliveira MM, Viegas SMF, Ferreira AP, Dias FCS. Eventos críticos na manutenção da conservação devacinas. Rev Enferm UFPE on line. [Internet]. 2018 [acessado em 29 dez. 2018];12(6):1781-9. Disponível em: https://periodicos.ufpe.br/revistas/revistaenfermagem/ article/view/230909/29231. https://doi.org/10.5205/19818963-v12i6a230909p1781-1789-2018.

29. Melo LTG, Coutinho RMC. Avaliação da prática de profissionais de enfermagem no processo de conservação de vacinas no município de Campinas. J Health Sci Inst [Internet]. 2018 [acessado em 29 dez. 2018];36(1):2833. Disponível em: https://www.unip.br/presencial/ comunicacao/publicacoes/ics/edicoes/2018/01 janmar/V36 n1 2018 p28a33.pdf.
30. Martins JRT, Alexandre BGP, Oliveira VC, Viegas SMF. Educação permanente em sala de vacina: qual a realidade? Rev Bras Enferm [Internet]. 2018 [acessado em 29 dez. 2018]71(Supl. 1):715-24. Disponível em: http://www.scielo.br/pdf/reben/v71s1/ pt 0034-7167-reben-71-s1-0668.pdf. http://dx.doi. org/10.1590/0034-7167-2017-0560.

31. Elisiário RN, Siman AG, Moreira TR, Carvalho CA, Amaro MOF. Avaliação das salas de vacinas nas unidades de estratégia de saúde da família. Enferm Rev [Internet]. 2017 [acessado em 29 dez. 2018];20(3):1-17. Disponível em: http://periodicos. pucminas.br/index.php/enfermagemrevista/article/ view/17228/13050. 